\title{
Technology Enhanced Learning with Limited Resources - Transforming Limitations into Advantages
}

\begin{abstract}
Medical education is rapidly changing, influenced by many factors including the changing healthcare environment, the changing role of the physician, altered societal expectations, rapidly changing medical science, and the diversity of pedagogical techniques. What would medicine be like in 20 years' time? Today's students will be practicing beyond the next 20 years and are expected and required to function effectively within everchanging health systems. What are the future challenges for medical education and how can we prepare to effectively face them?
\end{abstract}

Karunathilake, I.M.

The principal challenges in medical education can be categorized as changes occurring in the healthcare environment, the information explosion related to medicine and healthcare, and changes in the learner profile. Changes in the healthcare environment are driven by advances in technology, increasing costs, diversity of providers and settings, changing expectations of patients and the public (including professionalism, ethics and communication) and commodification of healthcare (Schuwirth \& van der Vleuten, 2006; Guze, 2015).

There is a global information explosion, which not only includes a rapid and exponential increase in published information but also the short- and long- term effects of this growth. The increasing quantum of knowledge in medicine no longer allows physicians to retain all knowledge that is necessary to provide quality patient care (Densen, 2011). Over 34000 references are added to MEDLINE each month from 4000 journals, and the doubling time of medical knowledge is estimated to be approximately 5 years. Knowledge is expanding faster than the ability to assimilate and apply it effectively; this is as true in education and patient care as it is in research.

Professor and Head,

Department of Medical Education,

Head, WHO Collaborating Centre for Medical Education,

Faculty of Medicine, University of Colombo, Sri Lanka.
Clearly, the simple inclusion of more material and/or time to the medical curriculum will not be an effective coping strategy. Therefore, a fundamental change in the approach to medical education has become imperative.

It is important to note that changes in the learner profile lead to challenges as well. Present day medical students are more technologically advanced than ever, and it is important for faculty to identify the inherent advantages as well as disadvantages faced by the "digital natives" who make up the majority. These changes in the learner profile lead to different approaches to learning, presenting ongoing challenges to medical educators (Beetham \& Sharpe, 2013).

These challenges will become even more pronounced for countries and institutions with limited by lack of financial, human and infrastructural resources. It is perceived that technological advances that have led to many changes in health systems and medical education are beyond the reach of countries with limited resources, especially in the South East Asian region, thus, the current situation will lead to and aggravate a vicious cycle.

Are we in South East Asia truly "lowresourced"? The South East Asian region has rich traditions in culture, healthcare and medical education. Availability of teaching materials such as patients and cadavers is much higher compared to other regions in the world. The high IT literacy, high accessibility to the internet, high connectivity due to widespread usage of mobile phones and social media is notable. Therefore, the term "Differently Resourced" may be more appropriate and positive in this context.

Can we use affordable and available technology to address and prepare for future challenges in medical education? There are many technologies currently being used in medical education including Simulation, Elearning/M-learning, Virtual Patients/Virtual communities, Virtual and Augmented Reality, Artificial Intelligence, and tools for sharing information and networking. 
Simulations have long been widely used for educational development. The aim of simulation is to imitate real patients, anatomic regions, or clinical tasks, and mirror the real-life circumstances in which medical services are rendered. Simulations can fulfil a number of educational goals. Even though simulation is perceived as high-cost, low-cost, low fidelity simulations can be as effective as high-end options.

E-learning and online learning can provide medical education with many affordable options. As an example, open source learning management system platforms such as MOODLE (Modular Object Oriented Dynamic Learning Environment) are relatively low cost yet used with high effectiveness in medical education. This technology can provide opportunities to change our approach in education to face future challenges. One example is the use of "flipped classrooms" in which students review an online lecture before the lecture session and come to the classroom to have an interactive session with the teacher.

Providing Continuous Professional Development (CPD) to the increasing numbers of health professionals working in diverse settings is a challenge for medical education. There are many opportunities offered through online learning for CPD driven by increasing access to the internet and increasing availability of IT facilities, and the evidence base for online learning for CPD is gaining strength. Access to information via the usage of repositories or digital libraries in medical education is on the rise. The Health Education Assets Library (HEAL) at $h$ ttp://www.healthcentral.org/and the Multimedia Educational Resource for Learning and Online teaching (MERLOT) at http://www.merlot.org/ are two examples for digital libraries. These are freely accessible resources of high quality information that benefit both medical educators and medical students.

Also Mobile learning (M Learning), offers many exciting opportunities for CPD. M learning refers to learning via a mobile device such as a tablet or smartphone. Such devices are ubiquitous among doctors and offer an array of potential opportunities for CPD. M Learning has progressed from e-mail and instant messaging services such as Yahoo, MSN, AOL, and
Skype, to more flexible and user friendly mobile applications such as WhatsApp and Viber for text, voice mode, video calling, as well as more sophisticated tools such as Articulate Studio for delivery of learning material and improvement of connectivity among learners in CPD (Masters et al., 2016).

Virtual Reality refers to the recreation of environments or objects as a complex, computer-generated image. In VR simulations, the computer display simulates the physical world and user interactions are with the computer within that simulated (virtual) world. Interaction can vary from looking around to interactively modifying the world. Augmented reality $(A R)$ involves adding a virtual experience to information obtained from the real environment. Even though most virtual reality initiatives are very sophisticated and of high cost, availability of smart phones and low-cost VR heat-sets provide opportunities for implementing virtual learning at a low cost.

In conclusion, technology offers many opportunities to overcome future challenges in medical education. Ignoring technology by citing it as unaffordable is no longer an option. Low-cost technology can be as effective as high cost options in achieving the desired educational outcomes. If you look in the right places, tomorrow's technology, at an affordable price, is already there.

\section{References}

Beetham, H. \& Sharpe, R. [Eds.] (2013) Rethinking pedagogy for a digital age: Designing for 21st century learning, Routledge.

Densen, P. (2011) Challenges and opportunities facing medical education, Transactions of the American Clinical and Climatological Association, 122, pp. 48-58.

Guze, P.A. (2015) Using Technology to Meet the Challenges of Medical Education, Transactions of the American Clinical and Climatological Association, 126, pp. 260-270.

Masters, K., Ellaway, R.H., Topps, D., Archibald, D. \& Hogue, R.J. (2016) Mobile technologies in medical education: AMEE Guide No. 105, Medical Teacher, 38, 6, pp.537-549.

Schuwirth, L.W. \& van der Vleuten, C.P. (2006) Medical education: Challenges for educationalists, BMJ: British Medical Journal, 333, 7567, p.544. 